\title{
Evaluation of the Predictive Value of Serum C-Reactive protein and Procalcitonin Levels in Early Detection of Anastomotic Leakage after Gastrointestinal Surgery
}

\author{
Mohamed A Elkerkary ${ }^{\star}$, Mahmoud Elnagar, Moustafa Abu Ali, and \\ Hamdy Shaban
}

Department of Surgery, Faculty of Medicine, Suez Canal university, Egypt

\begin{abstract}
Background: Anastomotic leak (AL) after gastrointestinal surgery is a serious postoperative complication that leads to significant morbidity and mortality. Objective: To evaluate the role of sequential postoperative serum determinations of C-reactive protein (CRP) and Procalcitonin (PCT) in the identification and prediction of anastomotic leakage after gastrointestinal surgery and to describe the kinetics of PCT and CRP after surgery and their relative usefulness for the early detection of anastomotic leaks after gastrointestinal surgery. Patients and Methods: This study is a clinical prospective randomized observational analytical cross sectional study conducted in the elective and emergency surgery theaters of the department of general surgery at Suez Canal university hospital from Jan 2015 to Jan 2016 on 45 patients who underwent small bowel, colonic and rectal surgery with resection and primary anastomosis. The data was collected by using a questionnaire and lab results. Results: In our study, $22.2 \%$ of study patients developed anastomotic leakage, $48.9 \%$ of the studied population was between the ages of (46-60) (range 18-72 years), and $62.2 \%$ of the study populations were female. Both biochemical markers were elevated in patients with anastomotic leakage at day2, 3 and 4 and showed slightly similar curves, percentages but the upper hand was for PCT than CRP in the point of early detection and in the much more sensitive and more specific. The Pearson correlation with fistula was more powerful for CRP than for PCT. Conclusion: Patients with PCT greater than $0.65 \mathrm{mg} / \mathrm{dL}$ and CRP greater than $21 \mathrm{mg} / \mathrm{dL}$ on Day4, even in the absence of clinical signs, are not permitted to leave the hospital and a diagnostic work-up for sepsis (lung, urinary tract, intra-abdominal, or wound) is actively pursued.
\end{abstract}

Key words: anastomotic leakage, Procalcitonin, $\mathrm{C}$ - reactive protein, gastrointestinal surgery

\section{Introduction}

Anastomotic leak $(\mathrm{AL})$ after gastrointestinal surgery is a serious postoperative complication that leads to significant morbidity and mortality. The frequency and consequences of anastomotic failure vary according to the site of anastomosis within the gastrointestinal tract. It is a serious complication after pancreatic surgery because dehiscence of anastomosis with autodigestion and destruction of surrounding tissue from leaking pancreatic juice is associated with a high mortality rate $^{(1)}$. 
Dehiscence after colorectal anastomosis increases the perioperative mortality rate due to peritonitis and septicemia, and adversely affects the late outcome in survivors because of increased local recurrence of carcinoma. Anastomotic leak may be used as an indicator of the quality of surgical care ${ }^{(1-2)}$. Anastomotic leakage is also responsible for delayed resumption of $\mathrm{Gl}$ transit, pain, prolonged hospital stay and increased costs. On the long term, it worsens functional prognosis after rectal surgery, promotes local recurrence and is a prognostic factor for poor one-year survival in patients undergoing cancer surgery ${ }^{(2-4)}$. According to the report of the French Association of Surgery, elective colorectal surgery in France has a mortality rate of about $4 \%$ and an overall morbidity of $35 \%(5-8)$. Anastomotic leakage is responsible for many of these complications, and accounts for one third of deaths ${ }^{(9-10)}$. The anastomotic leak rate varies from 1 to $40 \%$ in various reports depending on the definition $\operatorname{chosen}^{(7,8,11)}$. Despite the increased understanding about risk factors for anastomotic leakage and improvement in surgical technique, anastomotic leakage remains an important complication and occurs without obvious cause in some patients(11). According to Alves et al, the delayed diagnosis after Post-Operative Day 5(POD 5) of anastomotic leakage is associated with a mortality rate of $18 \%$, but minimal morbidity if diagnosed and treated before POD 5. Early detection of this complication is essential for timely institution of treatment, making early distinctive markers useful(5). Early detection allows appropriate treatment and less morbidity with a decreased need for ostomy, and less deterioration in the patients' quality of life. Clinical examination is often nonspecific, and symptoms of anastomotic leakage often develop late ${ }^{(12,13)}$. Since routine postoperative imaging is not efficient, the use of serum markers of inflammation has been proposed to aid in early detection of postoperative septic complications ${ }^{(14,16)}$. Thus, it has been shown that a serum C-reactive protein (CRP) greater than $12.5 \mathrm{mg} / \mathrm{dL}$ on the fourth postoperative day is predictive of septic complications. Moreover, fast track surgery and early rehabilitation have become standard in colorectal surgery with hospital discharge by the fifth postoperative day ${ }^{(17)}$. Anastomotic leaks and their consequences can occur well beyond that time, so it is necessary to find a marker of intra-abdominal infection with a high negative predictive value that allows safe hospital discharge with a low risk of re-admission $^{(18)}$. Procalcitonin (PCT) is an inflammatory marker with a short half-life. It is frequently used in the ICU as a prognostic marker for serious infections and has been used to evaluate the response to antibiotic treatment. However, PCT has not been adequately evaluated in the postoperative period $^{(19-23)}$. It is possible that the rise in PCT occurs earlier than that of CRP in bacterial infections ${ }^{(24-28)}$.

\section{Patients and Methods}

\section{Study design}

After approval of Ethics Committee of the Faculty of Medicine, Suez Canal University, this clinical prospective randomized study conducted in the elective and emergency surgery theaters of the department of general surgery at Suez Canal university hospital from Jan 2015 to Jan 2016 on 45 patients who underwent small bowel, colonic and rectal surgery with resection and primary anastomosis.

\section{Patients and Methods}

All patients were subjected to thorough medical history and physical examination, laboratory investigations to evaluate the patients general condition including; complete blood count, bleeding profile, electrolytes, arterial blood gases, serum albumin random blood sugar, liver and kidney 
function tests, Serum CRP and PCT. Pelviabdominal ultrasonography, Pelvi-abdominal computerized tomography with double contrast, Other studies as barium meal, enema, upper gastrointestinal endoscopy and colonoscopy if needed.

\section{Pre-operative preparation}

This included keeping the patient Nothing Per Oral (NPO), Intravenous fluids, Intravenous antibiotics, Mechanical preparation eitherpolyethylene glycol electrolyte solution, laxatives (mineral oil, agar and phenolphthalein),mannitol, enemas (water, $900 \mathrm{ml}$; glycerin, $100 \mathrm{ml}$ ), sodium picosulphate $10 \mathrm{mg}$, Bisacodyl (10 mg) + enemas, and diets low and non-residue. For each patient, the following data was recorded: surgical indication, For each intervention data was recorded concerning the surgical approach (laparotomy or laparoscopy), underlying pathology, type of resection (subtotal gastrectomy with gastro-jejunostomy, Roux en $\mathrm{Y}$ anastomosis, partial small bowel resection with entero-enteric anastomosis, right hemicolectomy, left hemicolectomy, total colectomy, anterior resection with colorectal anastomosis, proctocolectomy with ileoanal anastomosis, and hepaticojejunostomy anastomosis), characteristics of the anastomosis (mechanical or manual), presence or absence of drain. Patients were examined daily to assess the clinical condition (pain, fever, hemodynamic status, abdominal examination, return of bowel function, and any wound discharge, hemoglobin level). Serum CRP and PCT was measured on the day prior to surgery (Day 0 ) and on the first, second, third and fourth postoperative days (Day 1, Day 2 , Day 3 and Day 4) $)^{(26)}$. Anastomotic leakage was diagnosed on the clinical signs of peritonitis and/or clinical evidence of free faecal fluid within the abdomen or emerging from the drain site. On clinical suspicion, the diagnosis was confirmed by abdominal and pelvic $\mathrm{CT}$ scan, using intravenous and anorectal contrast. The patients were allocated into two groups according to the presence or absence of anastomotic leakage: with anastomotic leakage, without anastomotic leakage. The two groups were compared according to the following characteristics: gender and average age of patients, underlying pathology, planning of intervention (electives. urgent), surgical approach (laparotomy vs. laparoscopy), type of resection, mean hospital stay, postoperative morbidity and mortality, and mean values of serum CRP and Procalcitonin in the pre-and postoperative period. Serum CRP $<12 \mathrm{mg} / \mathrm{L}$ and serum Procalcitonin $<0.05 \mu \mathrm{g} / \mathrm{L}$ were considered normal values ${ }^{(27,28)}$. Inclusion criteria: adults ranging from 18-60 years old, both sexes included, Patients underwent elective or emergency gastrointestinal surgery with resection and primary anastomosis at the same setting while. Exclusion criteria: Patients with active infection (respiratory and urinary tract infection) before surgery or an acquired infection in the postoperative period other than leakage (respiratory, urinary tract and surgical wound infection), Patients those received chemotherapy and radiotherapy, Patients on long term corticosteroid therapy, Presence of a defunctioning stoma. After taking the blood samples, chemical analysis for all samples were done at once and value for each sample was recorded The blood samples ( $5 \mathrm{cc}$ of venous blood from peripheral vein) were collected and the process of serum separation were done by the researcher in the hospital laboratory then coded in the serum form and preserved in the freezer of the blood bank (of the hospital at $-80^{\circ} \mathrm{C}$ temperature) till the time of analysis by the specific kits using ELISA analysis in the hospital laboratory by a fixed laboratory technician. The disposable laboratory materials (e.g. blood sample tubes) used in the research will be brought by the researcher ${ }^{(26)}$. CRP was determined 
by immunonephelometry automated dimension Vista analyzer (Siemens, Erlangen, Germany). PCT was determined by homogeneous phase sandwich ELISA analysis (Brahms, Hennigsdorf, Germany) (26).

\section{Statistical analysis}

The process of data analysis was processed and encoded, these codes were entered into the computer through using statistical package for social science SPSS version 16 (SPSS Inc., Chicago, IL, USA). Quantitative data were expressed as means and standard deviation while qualitative data were expressed as numbers and percentages. Student $t$ test were used to test significance of difference for quantitative variables and Chi square were used to test significance of difference for qualitative variables. $p$-value $<0.05$ was considered statistically significant.

\section{Results}

In our study, $48.9 \%$ of the studied population was between the ages of (46-60) (range 18-72 years), 62.2\% were female, $46.7 \%$ of the patients had single co morbidity (table 1 ). $15.6 \%$ of our patients were mechanically prepared, $80 \%$ of patients were operated laparotomy and $20 \%$ of them were operated laparoscopically, $80 \%$ of study patients were manually hand sewn. While $17.7 \%$ of the study patients were stapled, $2.2 \%$ were mixed, drains were used in whole study patients, $20 \%$ of them were re explored, the mean of hospital stay days was 9.2 and the median was 7 days and it was between 3 to 35 days. The mean and the median value of serum albumin level of study patients were 2.9 and it ranged from 2.1 to 3.9 , the mean and the median value of serum hemoglobin level of study patients were 11.4 and it ranged from 9 to 14.8 patients were died which represents $17.8 \%$ (table 2 and 3). more sensitive and specific, the area under the ROC curve were slightly similar as at day 2, 3 it was more higher for PCT than CRP $0.820,0.959$ for $0.819,0.954$ and at day 4, it was higher for CRP than PCT 0.979 for 0.959. Also, serum PCT level at day 2 of 0.65 had sensitivity of $80 \%$ and specificity of $66 \%$. But serum CRP level at day 2 of 27 had the same sensitivity but more specific $80 \%$. At day 3, 4 and serum PCT levels of $0.7,0.65$ had $100 \%$ sensitivity and $95 \%$ specificity while that of serum CRP levels 24, 21 had lower sensitivity and specificity than PCT at day 3 as $90 \%, 89 \%$ and equal sensitivity at day 4 as $100 \%$ and more specific 98\% (Figures 1, 2) In comparison between PCT and CRP in the point of early detection, both markers were elevated in patients with anastomotic leakage at day2, 3 and 4 and showed slightly similar curves, percentages but the upper hand was for PCT than CRP in the point of early detection, higher sensitivity and specificity. The Pearson correlation with fistula was more powerful for CRP than for PCT (Figures 36).

\section{Discussion}

According to age distribution of the study population, $48.9 \%$ of the studied population was between the ages of (46-60) (range 18-72 years), The mean age of our patients was 47.3 with standard deviation 11.02 , while $50 \%$ of patients developed leakage was between the same age group and it's considered $11.1 \%$ of the whole patients. While in a pilot study conducted in France at the Digestive Surgery and Oncology Service, CHU de Dijon by Lagouttea, there were 100 patients with a mean age of 64 and a median age of 63 (range 20-87 years) ${ }^{(26)}$ where the median age for the patients in a study in Belgradeat Zvezdara University Clinical Centre by Scepanovic was $65^{(28-30)}$. While in a prospective study done in department of surgical oncology and colorectal surgery in poland and UK by Zawadzki, on 55 patients, the mean age 
was $66.1 \pm 11.2^{(30)}$. The mean age of nonleakage group in the study of Almeida, department of general surgery of Centrohospitalar de saojoao, Portugal, was 65.5 years, while in the leakage group was 69.5

Table 1: Demographic data and its relation to the anastomotic leakage

\begin{tabular}{|c|c|c|c|c|}
\hline \multirow{2}{*}{ Variables } & \multicolumn{2}{|c|}{ LEAK } & \multirow{2}{*}{ Total } & Percent \\
\cline { 2 - 5 } & No & Yes & & \\
\hline Age groups (Yrs.) & & & & \\
$\bullet \quad 18-30$ & 3 & 0 & 3 & 6.7 \\
$\bullet \quad 31-45$ & 12 & 4 & 16 & 35.6 \\
$\bullet \quad 46-60$ & 17 & 5 & 22 & 48.9 \\
$\bullet \quad>60$ & 3 & 1 & 4 & 8.9 \\
\hline Gender & & & & \\
$\bullet \quad$ Male & 15 & 2 & 17 & 37.8 \\
$\bullet \quad$ Female & 20 & 8 & 28 & 62.2 \\
$\bullet \quad$ Total & 35 & 10 & 45 & 100 \\
\hline
\end{tabular}

Table 4: Distribution of hemoglobin and albumin serum level characteristics

\begin{tabular}{|l|c|c|}
\hline & Albumin & Hemoglobin \\
\hline Mean & 2.913333 Eo & 11.49 \\
\hline Median & 2.900000 Eo & 11.00 \\
\hline Std. Deviation & .4424108 & 1.308 \\
\hline Minimum & 2.1000 & 9 \\
\hline Maximum & 3.9000 & 14 \\
\hline
\end{tabular}

While in the pilot study conducted at the Digestive Surgery and Oncology Service, CHU de Dijon by Lagouttea, 58 were men and 42 werewomen (26). And it was $57.1 \%$ males and $42.9 \%$ women in Scepanovic study ${ }^{(29)}$. While in Zawadzki study, 37 patients were males (67.3\%) and 18 patients were females $(32.7 \%)^{(30)}$. In the study of Almeida, 88 (50.8\%) of patients were males and $30(49.2 \%)$ were females ${ }^{(27)}$. Only $15.6 \%$ of our patients were mechanically prepared, $90 \%$ of patients developed leakage were not prepared. All patients in Lagouttea study, Scepanovic and Zawadzki study were mechanically prepared as these years ${ }^{(27)}$. In this study, $62.2 \%$ of the study populations were female, and $80 \%$ of patients who developed leakage were also female ( $17.7 \%$ of the total population).

Table 2: Surgical data characteristics and its relation to the anastomotic leakage

\begin{tabular}{|c|c|c|c|c|}
\hline \multirow{2}{*}{ Variables } & \multicolumn{2}{|c|}{ LEAK } & \multirow{2}{*}{ Total } & Percent \\
\cline { 2 - 5 } & No & Yes & & \\
\hline Approach & 7 & 2 & 9 & 20 \\
$\bullet \quad$ Laparoscopic & 28 & 8 & 36 & 80 \\
- Laparotomy & 35 & 10 & 45 & 100 \\
- Total & & & & \\
\hline Repair & & & & \\
- Mechanical & 6 & 2 & 8 & 17.8 \\
$\bullet \quad$ Manual & 28 & 8 & 36 & 80 \\
- Both & 1 & 0 & 1 & 2.2 \\
$\bullet \quad$ Total & 35 & 10 & 45 & 100 \\
\hline
\end{tabular}

studies were on elective cases ${ }^{(26,29,30)}$. Regarding the surgical technique of the operation, $80 \%$ of our patients were operated with laparotomy and 20\% were operated laparoscopically, and $80 \%$ of patients developed leakages were operated laparotomy. While in the pilot study at Dijon by Lagouttea, there were $65 \%$ of patients operated laparotomy and 35\% operated laparoscopically ${ }^{(26)}$. All patients in the study of Scepanovic were operated with laparotomy ${ }^{(29)}$. But at Zawadzki study, 29 patients (52.7\%) underwent a robotic surgery while the others (26 patients, $47.3 \%$ ) underwent open surgery(30). In the study of Almeida, there were $31(17.9 \%)$ patients were operated laparoscopically, one of them had developed leakage, while the others (142 (82.1\%) patients) were operated laparotomy, 23 patients of them had developed leakage ${ }^{(27)}$. In our study, $17.8 \%$ of patients suffered from perforated peptic, $15.6 \%$ of patients suffered from morbid obesity, $2.2 \%$ with esophageal cancer, $6.7 \%$ with cancer stomach, $4.4 \%$ with periampullary cancer, $6.7 \%$ cancer head of pancreas, $2.2 \%$ 
with cholangiocarcinoma,8.9\% with MVO, $4.4 \%$ with strangulated small bowel in hernia, $4.4 \%$ with cancer caecum, $2.2 \%$ with splenic flexure mass, $2.2 \%$ with recto sigmoid mass, $4.4 \%$ with cancer rectum, $4.4 \%$ with closure of colostomy, $2.2 \%$ with sigmoid volvulus $8.9 \%$ with ligated CBD post cholecystectomy, $2.2 \%$ with hepatic flexure mass. while $20 \%$ of patients developed leakage accounted for cancer head of pancreas, mesenteric vascular occlusion, morbid obesity and $10 \%$ of them accounted for stomach cancer, strangulated small bowel in Para umbilical hernia, cancer caecum, closure of Hartman's colostomy post recto sigmoid mass. While in the pilot study of

ROC Curve

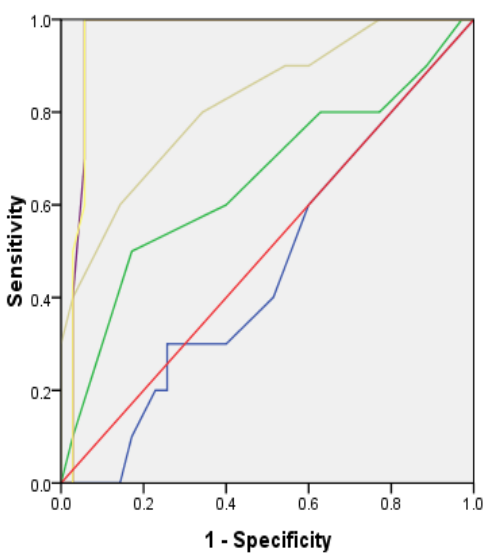

\begin{tabular}{|l|c|c|c|c|}
\hline \multicolumn{5}{|c|}{ Area Under the Curve } \\
\hline $\begin{array}{l}\text { Test Result } \\
\text { Variable(s) }\end{array}$ & Area & Cutoff & Sensitivity & Specificity \\
\hline PCTD0 & .461 & 0.02 & 40 & 49 \\
\hline PCTD1 & .651 & 0.65 & 60 & 60 \\
\hline PCTD2 & .820 & 0.65 & 80 & 66 \\
\hline PCTD3 & .959 & 0.7 & 100 & 95 \\
\hline PCTD4 & .959 & 0.65 & 100 & 95 \\
\hline
\end{tabular}

Figure 1: serum PCT level and characteristics for prediction of anastomotic leakage.

All patients in Zawadzki study were cancer colon ${ }^{(30)}$. In the study of Almeida, there were $129(74.5 \%)$ patients had cancer colon, 17 patients of them developed leakage, 8
Lagouttea, there was Cancer ( 52 patients): 2 rectum, 14 left colon, 16right colon, 1 transverse colon. Diverticulitis (17 patients). Benign colon tumor (3 patients). Re-anastomosis (8 patients). Others: polyps unresectable by endoscopy (7 patients), solitary ulcer (1 patient), rectal villous adenoma (1 patient),ileo-cecal stricture (2 patients), familial adenomatous polyposis ( 2 patients), anastomotic stricture (3 patients), migration of prosthetic material into the rectum ( 2 patients), ischemic stricture (1 patient), colonic metastasis from melanoma (1 patient) ${ }^{(26)}$. While it was $96.8 \%$ carcinoma in the study of Belgrade and was $3.2 \% \operatorname{IBD}^{(29)}$.

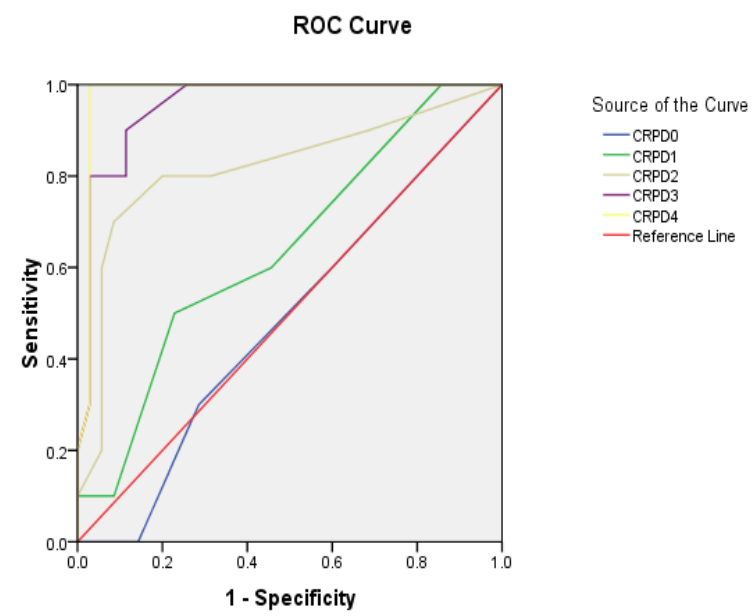

\begin{tabular}{|l|c|c|c|c|}
\hline \multicolumn{5}{|c|}{ Area Under the Curve } \\
\hline $\begin{array}{l}\text { Test Result } \\
\text { Variable(s) }\end{array}$ & Area & Cutoff & Sensitivity & Specificity \\
\hline CRPDo & .483 & 3 & 60 & 40 \\
\hline CRPD1 & .640 & 21 & 60 & 55 \\
\hline CRPD2 & .819 & 27 & 80 & 80 \\
\hline CRPD3 & .954 & 24 & 90 & 89 \\
\hline CRPD4 & .979 & 21 & 100 & 98 \\
\hline
\end{tabular}

Figure 2: Serum CRP levels and characteristics for prediction of anastomotic leakage.

(4.6\%) patients had IBD, 2 patients of them developed leakage, 4 (2.3\%) patients had diverticular disease, 32 (18.4\%) patients had other pathologies, 5 patients of them 
developed leakage ${ }^{(27)}$. In our study, $80 \%$ of our patients were manually hand sewn, $10 \%$ developed leakage. While $17.7 \%$ of our patients were stapled, $25 \%$ of them developed leakage and one patient with esophageal cancer was repaired mechanically for construction of gastric tube and manually for esophagogastric anastomosis. While Lagouttea and Belgrade there were 16\% protective ileostomy (in our study there were on protective stoma), $57 \%$ stapled anastomoses, and 43\% hand-sewn

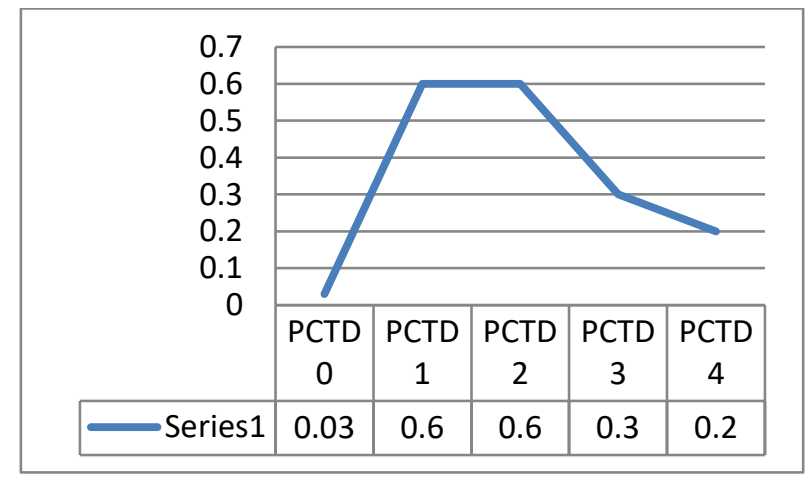

Figure 3: Median serum PCT levels at Do, D1, D2, D3, and D4 characteristics in non-anastomotic leak

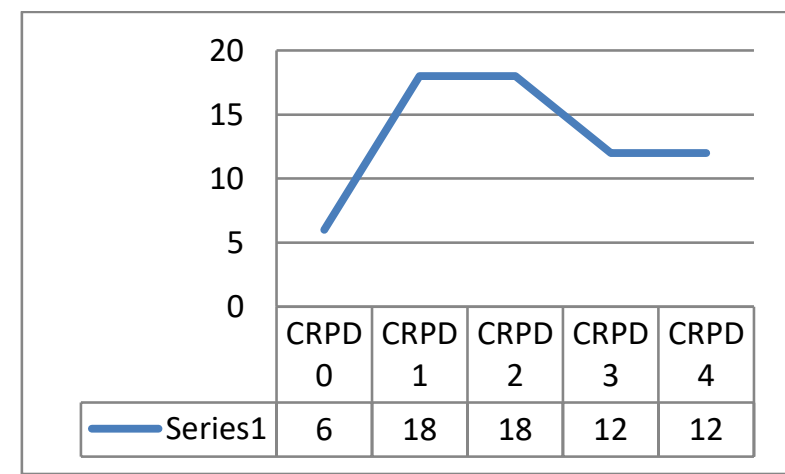

Figure 5: median serum CRP levels at Do, D1, D2, D3, and D4 characteristics in non-anastomotic leak

And it was $9.6 \%$ at the study done in Belgrade at Zvezdara University Clinical Centre by Scepanovic ${ }^{(29)}$. Also, it was 9.1\% (5 patients) at the study of Zawadzki and all of them were reexplored(30). In the study of Almeida, there were 24 (13.8) patients developed leakage, 21 of them had been re explored $^{(27)}$. The mean of hospital stay days was 9.2 and the median was 7 days and it was between 3 to 35 days. At anastomoses ${ }^{(26)}$. And it was $75 \%$ hand sewn versus $25 \%$ mechanical in the study of Scepanovic ${ }^{(29)}$. While in Zawadzki study, 29 patients (52.7\%) were stapled and 26 patients $(47.3 \%)$ were hand sewn(30). Drains were used in whole study patients while it was $62 \%$ in the pilot study of Lagouttea ${ }^{(26)}$. We found that $22.2 \%$ of our patients developed anastomotic leakage, $20 \%$ of them were re-explored. While it was $13 \%$ of patients included in pilot study done at Dijon by Lagouttea(26).

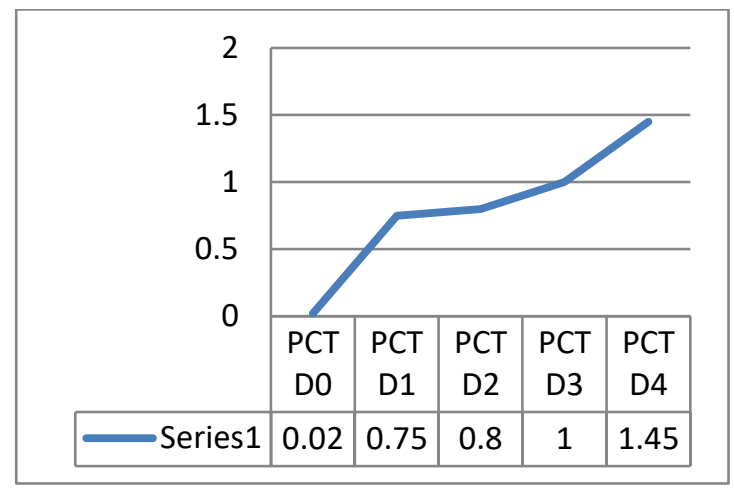

Figure 4: median serum PCT levels at Do, D1, D2, D3, and D4 characteristics in anastomotic leak

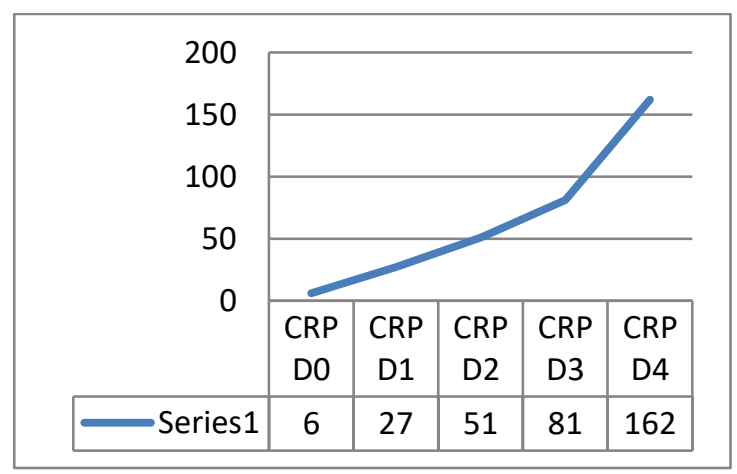

Figure 6: median serum CRP levels at Do, D1, D2, $D_{3}$, and D4 characteristics in anastomotic leak

Lagouttea study, the average length of stay was 12.5 \pm 9.8 days (range: 4-55 days)(26). While at Zawadzki study, the mean hospital stays in non AL was $5.9 \pm 1.6$, but in AL patients were $6.7 \pm 3.2$ days (30). In the study of Almeida, the mean hospital stay in non AL was 11.7 days, but in AL patients was 29.5 days ${ }^{(27)}$. The mean and the median value of serum albumin level of study patients were 2.9 and it ranged from 
2.1 to 3.9. The mean and the median value of serum hemoglobin level of study patients were 11.4 and it ranged from 9 to 14 . In this study 8 patients were died which represents $17.8 \%$, $50 \%$ of them had developed anastomotic leakage. At Lagouttea study, 2 patients died which represents 2 $\%(26)$. While 3 patients died in the study of Scepanovic Belgrade which represents 1.9 $\%(29)$. And no one died in Zawadzki study(30). According to the values of serum PCT levels, the median of Do, D1, D2, D3, and D4 were $0.3,0.6,0.6,0.3$ and 0.2 accordingly in the patients whom didn 't develop anastomotic leakage. While those who develop anastomotic leakage were 10 (22.2\%) patients as follow $0.2,0.75,0.8,1$ and 1.45 . At Lagouttea study, the values of serum PCT levels, the median of Do, D1, D2, D3, and D4 were $0.007,0.062,0.081,0.063$ and 0.045 in the patients whom didn't develop anastomotic leakage. While those who develop anastomotic leakage were $13(13 \%)$ patients as follow $0.013,0.163,0.134,0.111$ and $0.026^{(26)}$. At Zawadzki study, serum PCT were measured prior to surgery, $8 \mathrm{~h}$ after the incision, on the first and third postoperative day $(0,+8 \mathrm{~h}, \mathrm{POD} 1, \mathrm{POD} 3)$ and the values of median serum PCT levels were $0.05,0.07,0.72$ and 0.22 in the patients whom didn't develop anastomotic leakage. While those who develop anastomotic leakage were 5 (9.1\%) patients as follow $0.05,0.05,3.3$ and $4.88^{(30)}$. This study showed that serum PCT level of 0.02 at day o has no much significance in prediction of anastomotic leakage, sensitivity $40 \%$ and specificity $49 \%$ as well the serum PCT level of 0.65 at day 1 has no much significance, sensitivity $60 \%$ and specificity $60 \%$.This differs from day 2 as the sensitivity increased up to $80 \%$ with specificity $66 \%$ at the serum PCT level value of 0.65 , also at value of 0.75 sensitivity was $60 \%$ and specificity was $31 \%$ as the area under ROC curve increased 0.820 which showed a high significance for prediction of anastomotic leakage. While at day 3 and day 4 at the serum PCT level values of $0.7,0.65$, the sensitivity was $100 \%$ with specificity $95 \%$ and the area under ROC curve increased up to 0.959 which showed a very high significance for prediction of anastomotic leakage. The mean values of serum PCT levels increased on POD 1 and 3 in all patients, but the rise of PCT was significantly higher among the patients with AL only on POD 3.The mean PCT on POD 1 was $2.0 \mathrm{ng} / \mathrm{ml}$ in non-AL and 3.8 $\mathrm{ng} / \mathrm{ml}$ in AL patients, whereas on POD 3 it was $0.56 \mathrm{ng} / \mathrm{ml}$ and $10.4 \mathrm{ng} / \mathrm{ml}$, respectively(30). Analysis of ROC curves showed that PCT on POD 3 had AUC ofo.30. A cutoff value for PCT on POD 3 was calculated at the level of $3.26 \mathrm{ng} / \mathrm{ml}$ with $75 \%$ sensitivity and $100 \%$ specificity for $\mathrm{AL}^{(30)}$. According to the values of serum CRP levels, the median of Do, D1, D2, D3, and D4 were 6, 18, 18,12 and 12 in the patients whom didn't develop anastomotic leakage. While those who develop anastomotic leakage were 10 (22.2\%) patients as follow 6, 27, 51, 81 and 162. At Lagouttea study, the values of serum CRP levels, the median of Do, D1, D2, D3, and D4 were $0.47,12.4,15.2,11.30$ and 8.5 in the patients whom didn't develop anastomotic leakage. While those who develop anastomotic leakage were 13 (13\%) patients as follow 2.33, 15.5, 22.1, 23.05 and $20.8^{(26)}$. At Zawadzki study, serum CRP were measured prior to surgery, $8 \mathrm{~h}$ after the incision, on the first and third postoperative day $(0,+8 \mathrm{~h}, \mathrm{POD} 1, \mathrm{POD} 3)$ and the values of median serum CRP levels were $4.3,6.14,108.31$ and 105,26 in the patients whom did not develop anastomotic leakage. While those who develop anastomotic leakage were $5(9.1 \%)$ patients as follow 1.69, 4.4, 133.5 and $299.61{ }^{\text {(30). In the }}$ study of Almeida, serum CRP were measured preoperative and post-operative up to the gth day post-surgery. The values of mean serum CRP levels were15, 98, 132, $105,69,40,40,29,49$ and 36 in the patients whom didn`t had leakage. While those who 
develop anastomotic leakage were 24 (13.8\%) patients as follow 22, 116, 187, 201, 129, 162, 178, 201, 209 and 174 respectively ${ }^{(27)}$. This study showed that serum CRP level of 3 at day o has no much significance in prediction of anastomotic leakage, sensitivity $60 \%$ and specificity $40 \%$ as well the serum CRP level of 21 at day 1 has no much significance, sensitivity $60 \%$ and specificity $55 \%$. This differs from day 2 as the sensitivity increased up to $80 \%$ with specificity $80 \%$ at the serum CRP level value of 27 as the area under ROC curve increased 0.819 which showed a high significance for prediction of anastomotic leakage. While at day 3 and day 4 at the serum CRP level values of 24,21 the sensitivity was $90 \%, 100 \%$ with specificity $89 \%, 98 \%$ and the area under ROC curve increased up to $0.954,0.979$ which showed a very high significance for prediction of anastomotic leakage. In the study of Belgrade by Scepanovic, POD 2, mean serum CRP was $187 \mathrm{mg} / \mathrm{L}$ in leak group and132 mg/L in non leak group. On POD 3, those values were $201 \mathrm{mg} / \mathrm{L}$ in leak group and $105 \mathrm{mg} / \mathrm{L}$ in non-leak group. Based on the evaluation of the ROC curves, a cut-off value of $140 \mathrm{mg} / \mathrm{L}$ on POD 3 maximized the sensitivity (78\%) and specificity (31\%) of serum CRP in predicting the risk of leakage ${ }^{(29)}$. The mean value of CRP increased on POD 1 and POD 3 in all patients. The peak of CRP was significantly higher in the $A L$ group only on POD 3. On the third postoperative day the mean values of CRP were $114 \mathrm{mg} / \mathrm{l}$ in non-AL patients and 321 $\mathrm{mg} / \mathrm{L}$ in $\mathrm{Al}$ patients(30). Analysis of ROC curves showed that CRP on POD zhad AUC of 0.996 . A cut-off value for CRP on POD 3 was calculated at the level of $245.64 \mathrm{mg} / \mathrm{l}$, which resulted in100\% sensitivity and $98 \%$ specificity of $A^{(30)}$. There was no statistically significant difference in preoperative CRP between the non leakage and leakage group. In the postoperative period, however, it was clearly observed that from POD 2 onwards, the values of serum CRP were significantly higher in leakage group. In non-leakage group, mean serum CRP reached a peak on POD 2, followed by a rapid decline thereafter. On POD 2, mean serum CRP was $187 \mathrm{mg} / \mathrm{L}$ in leakage group and132 mg/L in non leakage group. On POD 3 , those values were $201 \mathrm{mg} / \mathrm{L}$ in leakage group and $105 \mathrm{mg} / \mathrm{L}$ in non leakage group. Based on the evaluation of the ROC curves a cut-off value of $140 \mathrm{mg} / \mathrm{L}$ on POD 3 maximized the sensitivity (78\%) and specificity (31\%) of serum CRP in predicting the risk of leakage ${ }^{(27)}$. In comparison between PCT and CRP in the point of early detection and the much more sensitive and specific, the area under the ROC curve were slightly similar as at day 2, 3 it was more higher for PCT than CRP $0.820,0.959$ for $0.819,0.954$ and at day 4, it was higher for CRP than PCT 0.979 for 0.959 . Also, serum PCT level at day 2 of 0.65 had sensitivity of $80 \%$ and specificity of $66 \%$. But serum CRP level at day 2 of 27 had the same sensitivity but more specific $80 \%$. At day 3,4 and serum PCT levels of $0.7,0.65$ had $100 \%$ sensitivity and $95 \%$ specificity while that of serum CRP levels 24, 21 had lower sensitivity and specificity than PCT at day 3 as $90 \%, 89 \%$ and equal sensitivity at day 4 as $100 \%$ and more specific $98 \%$. Indeed, both of the biochemical markers were elevated in patients with anastomotic leakage at day2, 3 and 4 and showed slightly similar curves, percentages but the upper hand is for PCT than CRP in the point of early detection and in the much more sensitive and more specific. At Lagouttea study, the area under the ROC curve for CRP was higher than that for PCT on Days 2, 3, and 4 as follow $0.711,0.801$ and 0.319 for $0.659, .674$ and 0.750 . Byanalysis of ROC curves for each marker on each day, sensitivity thresholds were established. At a threshold of 13.0 $\mathrm{mg} / \mathrm{dL}, \mathrm{CRP}$ had sensitivity and specificity of $80 \%$. With a threshold ofo.068 mg/dL, PCT had a sensitivity and specificity of $70 \%$ (maximum sensitivity obtained with 
the PCT $)^{(26)}$.Comparison of ROC curves for Belgrade patients was Day 3, Day 5, and Day 7 with respective area under the curve values of $0.738,0.756$ and $0.827^{(29)}$. The Pearson correlation with fistula was more powerful for CRP than for PCT in our study and in the study of Dijon. Thus, a patient with a PCT greater than $0.65 \mathrm{mg} / \mathrm{dL}$ and CRP greater than $21 \mathrm{mg} / \mathrm{dL}$ on Day4, even in the absence of clinical signs, is not permitted to leave the service and a diagnostic work-up for sepsis (lung, urinary tract, intraabdominal, or wound) is actively pursued. While in Lagouttea study it was PCT greater than $0.1 \mathrm{mg} / \mathrm{dL}$ and CRP greater than $12.5 \mathrm{mg} / \mathrm{dL}$. And it was on day 3, a cutoff value of CRP $135 \mathrm{mg} / \mathrm{l}$ was associated with the development of AL, providing a sensitivity of $73 \%$ and specificity of $73 \%$.on Day 7, a cutoff value of CRP $30 \mathrm{mg} / \mathrm{l}$ was associated with development of $A L$, providing a sensitivity of $80 \%$ and specificity of 90 $\%$, and a high diagnostic accuracy ${ }^{(26)}$.

\section{Conclusion}

Patients with $\mathrm{PCT}$ greater than $0.65 \mathrm{mg} / \mathrm{dL}$ and CRP greater than $21 \mathrm{mg} / \mathrm{dL}$ on day 4 , even in the absence of clinical signs, should not leave the hospital and a diagnostic work-up for sepsis (lung, urinary tract, intra-abdominal, or wound) should be actively pursued.

\section{References}

1. Bruce J, Krukowski H, Al-Khairy G, et al. Systematic review of the definition and measurement of anastomotic leak after gastrointestinal surgery. $\mathrm{Br} J$ Surg, 88, $1157-1168$.

2. Nesbokker A, Nygaard K, Lundi O. Outcome and late functional results after anastomotic leakage following mesorectal excision for rectal cancer. Br J Surg 2001; 88:400-4.

3. Greenblalt D, Weber S, O'Connor E, et al. Readmission after colectomy for cancer predictsone-year mortality. Ann Surg 2010; 251:659-69.

4. Mirnezami A, Mirnezami R, Chandrakumaran $\mathrm{K}$, et al. Increased local recurrence, and reduced survival from colorectal cancer following anastomotic leak: systematic review and meta analysis. Ann Surg 2011; 253:890-9.

5. Alves $A$, Panis $\mathrm{Y}$, Mathieu $\mathrm{P}$, et al. Postoperative mortality, and morbidity in French patients undergoing colorectal surgery. Arch Surg 2005; 140:278-26.

6. Platell C, Barwood N, Dorfmann G, et al. The incidence of anastomotic leaks in patients undergoing colorectal surgery. Colorectal Dis 2007; 9:71-9.

7. Alves A, Panis Y, Trancart D, et al. Factor associated with clinically significant anastomotic leakage after bowel resection: multivariate analysis of707 patients. World J Surg 2002; 26:499-502.

8. Bellows C, Webber L, Albo D, et al. Early predictors of anastomotic leaks after colectomy. Tech Coloproctol 2009; 13:41-7.

9. Marra F, Steffer T, Kalak N, et al. Anastomotic leakage as a risk factor for the long-term outcome after curative resection of colon cancer. Eur J Surg Oncol 2009; 35:1060-4.

10. Kingham T, Pachter H. Colonic anastomosis leak: risk factors, diagnosis, and treatement. J Am Coll Surg 2009; 208:269-78.

11. Matthiessen $P$, Henriksson $M$, Hallbook $O$, et al. Increase of serum C-reactive protein is an early indicator of subsequent symptomatic anastomotic leakage after anterior resection. Colorectal Dis 2008; 10:75e80.

12. Karliczek A, Harlaar N, Zeebregts C, et al. Surgeons lack predictive accuracy for anastomotic leakage in gastro-intestinal surgery. Int J Colorectal Dis 2009; 24:569-76.

13. Hyman N, Manchester T, Osler T, et al. Anastomotic leaks after intestinal anastomosis: it's later than you think. Ann Surg 2007; 245:254-8.

14. Ortega P, Radais F, Facy O, et al. C-reactive protein is an early predictor of 
septic complications after elective colorectal surgery. World J Surg 2010; 34:808-14.

15. Korner H, Nielsen H, Soreide J, et al. Diagnostic accuracy of $\mathrm{C}$ reactive protein for intra-abdominal infections after colorectal resections. J Gastrointest Surg 2009; 13:1599-606.

16. Woeste $G$, Muller C, Bechstein W, et al. Increasesed serum levels of C-reactive protein precede anastomotic leakage in colorectal surgery. World J Surg 2010; 34:140-6.

17. Kehlet H, Wilmore D. Evidence-based surgical care and the evolution of fast track surgery. Ann Surg 2008; 248:18998.

18. Charles P, Tinel C, Barbar S, et al.Procalcitonin kinetics within the first days of sepsis: relationship with the appropriateness of antibiotic therapy and the outcome. Crit Carezoog; 13: R38.

19. Rau B, Frigerio I, Buchler M, et al. Evaluation of Procalcitonin for predicting septic multi organ failure and overall prognosis in secondary peritonitis: a prospective, international multicenter study. Arch Surg2007; 36:1147-54.

20. Novotny A, Emmanuel K, Matevossian $E$, et al. Use of Procalcitonin for early prediction of lethal outcome of postoperative sepsis. Am J Surg2007; 194:35-9.

21. Reith H, Mittelkotter U, Debus E, et al. Procalcitonin in early detection of postoperative complications. Dig Surg 1998; 15:260-5.

22. Chromik A, Endter F, Uhl W, et al. Preemptive antibiotic treatment vs 'standard' treatment in patients with elevated serum procalcitonin levels after elective colorectal surgery: a prospective randomized pilot study. Langenbecks Arch Surg 2006; 391:18794.

23. Dandona P, Nix D, Wilson M, et al. Procalcitonin increase after endotoxin injections in normal subjects. J Clin Endocrinol Metab 1994; 79:1605-8.

24. Gendrel D, Assicot M, Raymond J, et al. Procalcitonin as a marker for early diagnosis of neonatal infection. J Pediatric 1996; 128:570-3.

25. Gendrel D, Raymond J, Assicot M, et al. Measurement of Procalcitonin levels in children with bacterial or viral meningitis. Clin Infect Dis997; 24:1240-2.

26. Lagouttea N, Facya O, Ravoirea A, et al. $C$-reactive protein and procalcitonin for the early detection of anastomotic leakage after elective colorectal surgery: Pilot study in 100 patients. Journal of Visceral Surgery (2012) 149, e345e349.

27. Almeida A, Faria G, Moreira H, et al. Elevated serum C-reactive protein as a predictive factor for anastomotic leakage in colorectal surgery. I Journal of Surgery 10 (2012) 87e91.

28. Joram N, Muller J, Denizot S, et al. Umbilical cord blood procalcitonin level in early neonatal infections: a 4-year university hospital cohort study. Eur J Clin Microbiol Infect Dis 2011; 30:1005-1013.

29. Scepanovic MS, Kovacevic B, Cijan V. Creactive protein as an early predictor for anastomotic leakage in elective abdominal surgery, Tech Coloproctol (2013) 17:541-547.

30. Zawadzki M, Roman M, Marek R, et al. C-reactive protein and procalcitonin predict anastomotic leaks following colorectal cancer resections - a prospective study, Videosurgery Miniinv 2015; 10 (4): 567-573. 